\title{
A REACTION-DIFFUSION SYSTEM ARISING IN MODELLING MAN-ENVIRONMENT DISEASES*
}

\author{
BY \\ V. CAPASSO (Università di Bari, Bari, Italy) \\ AND \\ K. KUNISCH (Technische Universität Graz, Graz, Austria)
}

1. Introduction. In this paper a reaction-diffusion system is analyzed based on a parabolic equation coupled with a nonlinear ordinary differential equation via a boundary feedback integral operator. The system is written in the following form:

$$
\begin{aligned}
& \frac{\partial}{\partial t} v_{1}(t, x)=\Delta v_{1}(t, x)-a_{1} v_{1}(t, x), \\
& \frac{\partial}{\partial t} v_{2}(t, x)=-a_{2} v_{2}(t, x)+g\left(v_{1}(t, x)\right),
\end{aligned}
$$

in $(0, \infty) \times \Omega$, subject to the following boundary condition:

$$
\frac{\partial}{\partial n} v_{1}(t, x)+\beta v_{1}(t, x)=\int_{\Omega} K\left(x, x^{\prime}\right) v_{2}\left(t, x^{\prime}\right) d x^{\prime},
$$

on $(0, \infty) \times \Gamma$ and also subject to suitable initial conditions. Here $\Omega$ denotes an open bounded domain in $\mathbf{R}^{n}(n=2,3)$ with boundary $\Gamma$. As usual, $\Delta$ stands for the Laplace operator and $\partial / \partial n$ denotes the outward normal derivative on $\Gamma$.

The motivation for studying (1.1), (1.1b) comes from the fact that it serves as a model for a class of man-environment epidemic systems when we adopt the following interpretation. We consider $\Omega$ as the habitat (environment) in which the human population is exposed to an infectious agent, so that an epidemic phenomenon arises because the infected human population acts as a multiplier of the infectious agent itself. In this case $v_{1}(t, x)$ denotes the concentration of the infectious agent at a point $x \in \Omega$ and time $t \geq 0$, while $v_{2}(t, x)$ denotes the spatial density of the human infective population. In this context $(1.1),(1.1 \mathrm{~b})$ is a more realistic generalization of a previous model proposed by V. C. and co-workers $[6,8]$ to describe fecal-orally transmitted diseases (cholera, typhoid fever, infectious hepatitis, etc.) which are typical for the European Mediterranean regions. For this kind of epidemic the infectious agent is multiplied by the infective human population and then sent to the sea through the sewage; because of the peculiar eating habits of the population of these regions the agent may return via some diffusion-transport mechanism to any point of the habitat $\Omega$, where the infection process is restarted. Thus the kernel in $(1.1 \mathrm{~b})$ mathematically 
describes the transfer mechanism of the infectious agent generated by the human population at $x^{\prime} \in \Omega$ to the point $x \in \Gamma$. Clearly, then, the boundary may be split into two disjoint parts: $\Gamma_{1}$, denoting the seashore at which $K(x, \cdot) \geq 0$, and its complement $\Gamma_{2}=\Gamma-\Gamma_{1}$, at which $K(x, \cdot)$ may be taken identically zero. The Laplace operator takes into account a simplified random dispersal of the infectious agent in the habitat $\Omega$. Finally, $g\left(v_{1}(t, x)\right)$ denotes the force of infection of the human population due to the infectious agent at $(t, x) \in(0, \infty) \times \Omega$. The parameters $a_{1}$ and $a_{2}$ are intrinsic decay parameters of the two populations. We also observe that without coupling on the boundary the two populations will both tend to extinction if $a_{1}$ and $a_{2}$ are positive.

This model was proposed for the first time in [5], where diffusion of the infective population was also considered in order to keep the compactness of the solution operator of the system. Later in [7] an analogous system was studied with slow diffusion $\left(\Delta v_{1}^{m}\right)$ for the infectious agent and linear diffusion $\left(\Delta v_{2}\right)$ for the human infectives. In both cases the asymptotic behavior of classical solutions was analyzed in order to obtain threshold theorems for the extinction of the epidemic.

In this paper we study system (1.1), (1.1b) where the second equation is an ordinary differential equation, thus losing compactness of the solution operator. Actually, the particular structure of the system, in which the coupling of the two equations occurs via a boundary feedback integral operator, does not allow us to refer to classical results when considering well-posedness or qualitative properties of (1.1), (1.1b).

In Sec. 2 we study existence and uniqueness of (1.1), (1.1b) and give conditions which guarantee that the solution operator associated with the system generates a strongly continuous semigroup of nonlinear operators on the space of $L^{1}(\Omega) \times L^{1}(\Omega)$ as well as $C(\bar{\Omega}) \times C(\bar{\Omega})$ functions. Under natural assumptions these semigroups are shown to be positivity preserving with respect to the natural cones in these spaces. Section 3 is devoted to some aspects of the linearized model and contains a density result which is essential for the proof of positivity of the solution semigroup. Finally, in Sec. 4 we continue to investigate qualitative properties of the solutions of (1.1), $(1.1 \mathrm{~b})$ and, in particular, we show monotonicity as well as concavity properties.

Since the completion of this manuscript several additional questions associated with modelling aspects of system (1.1), (1.1b) have been addressed. An optimal control problem arises if one wishes to reduce the epidemic phenomenon described by the above model by diminishing the boundary feedback along the seashore using a sewage treatment program (see [16]). In the actual modelling problem one has data of the epidemic and one has made a choice of using (1.1), (1.1b) as a model equation. There remains the problem of fixing the values of the coefficients in (1.1), $(1.1 \mathrm{~b})$, most importantly the strength of the feedback operator which is determined by $K$. This parameter estimation problem is addressed in $[17,18]$.

2. Existence, uniqueness, and regularity. In this section we show that unique solutions of the special reaction-diffusion system that is studied in this paper exist and that they generate nonlinear positivity-preserving $C_{0}$-semigroups on $L^{1}(\Omega) \times L^{1}(\Omega)$ and on $C(\bar{\Omega}) \times C(\bar{\Omega})$. Further regularity of the solutions is studied. Let us first repeat 
the system that is under consideration:

$$
\begin{aligned}
\frac{\partial v_{1}}{\partial t} & =-a_{1} v_{1}+\Delta v_{1}, \\
\frac{\partial v_{2}}{\partial t} & =-a_{2} v_{2}+g\left(v_{1}\right), \quad \text { for }(t, x) \in(0, \infty) \times \Omega, \\
\frac{\partial v_{1}}{\partial n}+\beta v_{1} & =\int_{\Omega} K\left(x, x^{\prime}\right) v_{2}\left(t, x^{\prime}\right) d x^{\prime}, \quad(t, x) \in(0, \infty) \times \Gamma, \\
v_{1}(0, x) & =v_{1}^{0}(x), \quad v_{2}(0, x)=v_{2}^{0}(x) .
\end{aligned}
$$

Here $\Omega$ is a bounded domain (in $\mathbf{R}^{n}$ ) with boundary $\Gamma$. As usual, $\partial / \partial n$ denotes the outward normal derivative. Two sets of regularity conditions will be used for the parameters $\left(a_{1}, a_{2}, \Omega, \beta, g, K\right)$ determining (2.1). In the notation of these requirements we follow [15]

$$
\begin{aligned}
& a_{1} \in L^{\infty}(\Omega), \quad a_{1}(x) \geq \alpha_{1} \quad \text { a.e. for some } \alpha_{1}>0, \\
& \Gamma \text { is } C^{0,1}-\text { smooth (Lipschitz continuous boundary), } \\
& \beta \in L^{\infty}(\Gamma), \quad \beta \geq 0, \\
& K \in L^{\infty}(\Gamma \times \Omega),
\end{aligned}
$$

and

$$
\begin{aligned}
& a_{1} \in C^{1}(\bar{\Omega}), \quad a_{1}(x) \geq \alpha_{1}>0, \quad x \in \Omega, \\
& a_{2} \in C(\bar{\Omega}), \quad a_{2} \geq 0,
\end{aligned}
$$

$\Gamma$ is $C^{2.1}-$ smooth (second derivatives Lipschitz continuous),

$\beta \in C^{1}(\Gamma), \quad \beta \geq 0$,

$g$ is globally Lipschitz continuous from $L^{1}(\Omega)$ to $L^{1}(\Omega)$ with Lipschitz norm $\|g\|$, $K \in L^{\infty}(\Gamma \times \Omega), \quad K_{x} \in L^{\infty}(\Gamma \times \Omega)$.

We shall not distinguish between the mapping $g$ and the substitution operator $g: L^{1}(\Omega) \rightarrow L^{1}(\Omega)$ given by $(g \varphi)(x)=g(\varphi(x))$. The requirement on $g$ is satisfied if $|g(x)| \leq \tilde{\beta}+\tilde{\alpha}|x|$, for some $\tilde{\beta} \geq 0, \tilde{\alpha} \geq 0$ [12, p. 161].

We start our analysis by a formal homogenization of the boundary condition $(2.1 \mathrm{c})$. Consider for $v_{2} \in C\left(0, T ; L^{1}(\Omega)\right), T>0$ and $t>0$, the elliptic equation

$$
\begin{gathered}
\Delta \tilde{w}(t, \cdot)-a_{1} \tilde{w}(t, \cdot)=0, \quad \text { on } \Omega, \\
B \tilde{w}(t, \cdot)=\Phi\left(v_{2}(t, \cdot)\right), \quad \text { for } t>0 \text { on } \Omega,
\end{gathered}
$$

where $B \psi=\partial \psi / \partial n+\left.\beta \psi\right|_{\Gamma}$ and $\Phi(\psi)(x)=\int_{\Omega} K\left(x, x^{\prime}\right) \psi\left(x^{\prime}\right) d x^{\prime}$, for $\psi \in L^{1}(\Omega)$, $x \in \Gamma$. The solution of $(2.2)$ is denoted by $\tilde{w}$ or $\tilde{w}(t), \tilde{w}(t, x)$, or $\tilde{w}\left(t, v_{2}\right)$. We put

$$
v_{1}=w+\tilde{w},
$$

so that $\partial v_{1} / \partial t=\partial w / \partial t+\partial \tilde{w} / \partial t$ and $\Delta v_{1}(t, \cdot)=\Delta w(t, \cdot)+\Delta \tilde{w}(t, \cdot)$. Thus $(2.1)$ is formally equivalent to

$$
\begin{aligned}
\frac{\partial}{\partial t} w(t) & =\Delta w-a_{1} w-\frac{\partial}{\partial t} \tilde{w}, \quad \text { for } t>0 \text { on } \Omega, \\
B w(t) & =0 \\
w(0, \cdot) & =v_{1}^{0}-\tilde{w}(0),
\end{aligned}
$$




$$
\begin{aligned}
\frac{\partial}{\partial t} v_{2}(t) & =-a_{2} v_{2}+g(w+\tilde{w}), \quad \text { for } t>0 \text { on } \Omega, \\
v_{2}(0) & =v_{2}^{0},
\end{aligned}
$$

and

$$
\begin{array}{cl}
\Delta \tilde{w}(t)-a_{1} \tilde{w}(t)=0, & \text { for } t \geq 0 \text { on } \Omega, \\
B \tilde{w}(t)=\Phi\left(v_{2}(t)\right), & \text { for } t \geq 0 \text { on } \Gamma .
\end{array}
$$

We summarize some properties concerning the boundary value problem of the third kind arising in (2.6). For convenience we also recall that by Green's formula:

$$
\sum_{i=1}^{n} \int_{\Omega} \frac{\partial u}{\partial x_{i}} \frac{\partial \psi}{\partial x_{i}} d x+\int_{\Omega} a_{1} u \psi d x=\int_{\Omega}\left(-\Delta+a_{1}\right) u \psi d x+\int_{\Gamma} \frac{\partial u}{\partial n} \psi d \sigma
$$

for all $u \in H^{2}(\Omega), \psi \in H^{1}(\Omega)$, if $\Gamma$ is $C^{2,1}$-smooth.

LEMMA 2.1. Let the assumption of (A1) hold for $\Omega, a_{1}$, and $\beta$ and let $h \in L^{2}(\Gamma)$. Then there exists a weak solution $u \in H^{1}(\Omega)$ of

$$
\Delta u-a_{1} u=0 \quad \text { on } \Omega, \quad B u=h \quad \text { on } \Gamma
$$

this means

$$
\int_{\Omega} \sum_{i=1}^{n} \frac{\partial u}{\partial x_{i}} \frac{\partial \psi}{\partial x_{i}} d x+\int_{\Omega} a_{1} u \psi d x+\int_{\Gamma} \beta u \psi d x=\int_{\Gamma} h \psi d x
$$

for every $\psi \in L^{1}(\Omega)$. Moreover,

$$
|u|_{H^{\prime}(\Omega)} \leq C_{1}\left(\Omega, a_{1}, \beta\right)|h|_{L^{2}(\Gamma)} .
$$

If the assumptions of (A2) hold for $\Omega, a_{1}$, and $\beta$, and if $h \in H^{1 / 2}(\Gamma)$, then $u \in H^{2}(\Omega), u$ satisfies $(2.7)$, and

$$
|u|_{H^{2}(\Omega)} \leq C_{2}\left(\Omega, a_{1}, \beta\right)|h|_{H^{1 / 2}(\Gamma)} .
$$

Proof. Using the trace theorem (see, e.g., [15, p. 130]) and the Lax-Milgram theorem, the proof of the first part is quite standard. The regularity result of the second part can be verified employing [15, Theorem 21.1], for example; see also [15, p. 338]. For given $v_{2}$ we call $\tilde{w} \in L^{1}\left(0, T ; H^{1}(\Omega)\right)$ a weak solution of $(2.6)$ on $[0, T]$ if

$$
\int_{\Omega} \sum_{i=1}^{n} \frac{\partial \tilde{w}(t)}{\partial x_{i}} \frac{\partial \psi}{\partial x_{i}} d x+\int_{\Omega} a_{1} \tilde{w}(t) \psi d x+\int_{\Gamma} \beta \tilde{w}(t) \psi d x=\int_{\Gamma} \Phi\left(v_{2}(t)\right) \psi d x
$$

for all $\psi \in H^{1}(\Omega), t \in[0, T]$.

LemMA 2.2. Let $(\mathrm{A} 1)$ hold and let $v_{2} \in W^{1.1}\left(0, T ; L^{1}(\Omega)\right), T>0$, be given. Then a unique weak solution $\tilde{w}=\tilde{w}\left(v_{2}\right)$ of $(2.6)$ exists, it satisfies $\tilde{w} \in W^{1.1}\left(0, T ; H^{1}(\Omega)\right)$, and $\dot{\tilde{w}}$ is the weak solution of $(2.6)$ with right-hand side $\Phi\left(\dot{v}_{2}\right)$. Further, there exists a constant $C$ independent of $t \in[0, T]$ and $v_{2}, \bar{v}_{2} \in W^{1.1}\left(0, T ; L^{1}(\Omega)\right)$ such that

$$
\left|\dot{\tilde{w}}\left(t, v_{2}\right)-\dot{\tilde{w}}\left(t, \bar{v}_{2}\right)\right|_{H^{\prime}(\Omega)} \leq C\left|\dot{v}_{2}(t)-\dot{\bar{v}}_{2}(t)\right|_{L^{\prime}(\Omega)} \quad \text { for a.e. } t \in[0, T]
$$


If moreover (A2) holds, then $\tilde{w}=\tilde{w}\left(v_{2}\right) \in W^{1,1}\left(0, T ; H^{2}(\Omega)\right), \tilde{w}$ satisfies the boundary condition in (2.6), and for some constant $\tilde{C}$ independent of $t \in[0, T]$ and $v_{2}$, $\bar{v}_{2} \in W^{1,1}\left(0, T ; L^{1}(\Omega)\right)$ we have

$$
\left|\dot{\tilde{w}}\left(t, v_{2}\right)-\dot{\tilde{w}}\left(t, \bar{v}_{2}\right)\right|_{H^{2}(\Omega)} \leq \widetilde{C}\left|\dot{v}_{2}(t)-\dot{\bar{v}}_{2}(t)\right|_{L^{1}(\Omega)} \quad \text { for a.e. } t \in[0, T] .
$$

The space $W^{1,1}\left(0, T ; L^{1}(\Omega)\right)$ can be replaced by $C^{1}\left(0, T ; L^{1}(\Omega)\right)$ in all the above statements. Moreover, if $v_{2} \in C^{1}\left(0, T ; L^{1}(\Omega)\right)$ and (A2) holds, then $\dot{\tilde{w}}$ satisfies the boundary condition in (2.6) with $\Phi\left(v_{2}\right)$ replaced by $\Phi\left(\dot{v}_{2}\right)$ for every $t \geq 0$.

Proof. Let $v_{2} \in W^{1,1}\left(0, T ; L^{1}(\Omega)\right)$. By Lemma 2.1 a weak solution $\tilde{w}\left(v_{2}\right)$ of $(2.6)$ exists and $\tilde{w}\left(v_{2}\right) \in C\left(0, T ; L^{1}(\Omega)\right)$. Similarly, let $z \in L^{1}\left(0, T ; H^{1}(\Omega)\right)$ be the weak solution of $(2.6)$ with $\Phi\left(v_{2}\right)$ replaced by $\Phi\left(\dot{v}_{2}\right)$. We will show that $z=\dot{\tilde{w}}$. For $t \in[0, T]$ and $t+\tau \in[0, T]$ we obtain, using (2.8) and (A1),

$$
\left|\frac{\tilde{w}(t+\tau)-\tilde{w}(t)}{\tau}-z\right|_{H^{\prime}(\Omega)} \leq C_{1}|K|_{L^{\infty}} \cdot \sqrt{l}\left|\frac{v_{2}(t+\tau)-v_{2}(t)}{\tau}-\dot{v}_{2}(t)\right|_{L^{\prime}(\Omega)},
$$

where $l=\int_{\Gamma} d s$. We can take a.e. the limit $\tau \rightarrow 0$ in the above inequality to obtain $\dot{\tilde{w}}=z$ a.e. on $[0, T]$ and thus $\tilde{w} \in W^{1,1}\left(0, T ; H^{1}(\Omega)\right)$. If $v_{2} \in C^{1}\left(0, T ; L^{1}(\Omega)\right)$, then $z \in C\left(0, T ; H^{1}(\Omega)\right)$ and thus $\tilde{w} \in C^{1}\left(0, T ; H^{1}(\Omega)\right)$.

Since $\dot{\tilde{w}}\left(v_{2}\right)-\dot{\tilde{w}}\left(\bar{v}_{2}\right)$ is the weak solution of (2.6) with right-hand side $\Phi\left(\dot{v}_{2}\right)-\Phi\left(\dot{\bar{v}}_{2}\right)$, we have (2.10) with $C=C_{1} \sqrt{l}|K|_{L^{\infty}(\Gamma \times \Omega)}$. Next, we add assumption (A2). Then $\tilde{w}\left(v_{2}\right) \in W^{1,1}\left(0, T ; H^{2}(\Omega)\right)$ and $(2.11)$ holds. This follows from the second part of Lemma 2.1, specifically (2.9) and the observation that $\left|\Phi v_{2}(t)\right|_{H^{1 / 2}(\Gamma)} \leq\left(|K|_{L^{\infty}(\Gamma \times \Omega)}+\right.$ $\left.\left|K_{x}\right|_{L^{\infty}(\Gamma \times \Omega)}\right)$. The remaining assertions can easily be verified and the proof is complete.

We now prepare some semigroup theoretic results. Let $\widetilde{A}_{1}$ be the operator defined by

$$
\begin{aligned}
D\left(\tilde{A}_{1}\right) & =\left\{v \in H^{2}(\Omega) \mid B v=0\right\}, \\
\tilde{A}_{1} v & =\Delta v-a_{1} v,
\end{aligned}
$$

and denote by $A_{1}$ the closure of $\tilde{A}_{1}$ in $L^{1}(\Omega)$. If (A2) holds, then by the trace theorem (e.g., [15, p. 130]) $B v \in H^{1 / 2}(\Gamma)$ for $v \in D\left(\widetilde{A}_{1}\right)$. Note that the closure of $\widetilde{A}_{1}$ in $L^{1}(\Omega)$ exists; for if $u_{j} \rightarrow 0$ in $L^{1}(\Omega), u_{j} \in D\left(A_{1}\right)$, and $\tilde{A}_{1} u_{j} \rightarrow v$ in $L^{1}(\Omega)$, then for all $w \in C_{0}^{\infty}\left(C^{\infty}\right.$-functions with compact support $)$ we have by Green's formula [15, $\mathrm{p}$. 336]

$$
\int_{\Omega} v w d x=\lim _{j} \int_{\Omega} \tilde{A}_{1} u_{j} w d x=\lim _{j} \int_{\Omega} u_{j} \tilde{A}_{1} w d x=0,
$$

and thus $v=0$. This implies closeability of $\tilde{A}_{1}$. Here we used $C^{2.1}$-smoothness of $\Gamma$. For a characterization of $D\left(A_{1}\right)$ in the case of Dirichlet boundary conditions see [ 10 , p. 251]. For our case of boundary conditions of the third type a characterization of $D\left(A_{1}\right)$ seems to be difficult to obtain.

Further, let $A_{c}$ be the operator given by

$$
\begin{aligned}
D\left(A_{C}\right) & =\left\{v \in C(\bar{\Omega}) \cap H^{2}(\Omega) \mid B v=0, \Delta v-a_{1} v \in C(\bar{\Omega})\right\}, \\
A_{c} v & =\Delta v-a_{1} v .
\end{aligned}
$$


Lemma 2.3. Let (A2) hold. Then $A_{c}$ generates a positive, analytic, and compact semigroup $S_{c}(t)$ on $C(\bar{\Omega})$. Similarly, $A_{1}$ generates a positive, analytic semigroup $S_{1}(t)$ on $L^{1}(\Omega)$.

Proof. The first claim is verified in [1, Theorem 8.2], for example. The second assertion is essentially contained in [10] under stronger regularity assumptions on $\Gamma$. We prefer to give an independent proof here, following a technique described in [13].

Recall that

$$
|v|_{L^{\prime}}=\sup \left\{\left.\int_{\Omega} v \varphi d x\left|\varphi \in C_{0}^{\infty}(\Omega),\right| \varphi\right|_{L^{\infty}} \leq 1\right\},
$$

[13, p. 218]. Analyticity of $S_{c}(t)$ implies the existence of constants $R \geq 0, M>0$, $\theta \in(0, \pi / 2)$ such that

$$
\left\|\left(\lambda I-A_{C}\right)^{-1}\right\|_{L^{\infty}} \leq M|\lambda|^{-1},
$$

for all $\lambda \in \Sigma_{\theta}=\{\mu \in \mathbf{C}:|\arg \mu|>\theta,|\mu| \geq R\}$. Since $C_{0}^{\infty}$ is contained in the range of $\lambda I-A_{c}$ for $\lambda \in \Sigma_{\theta}$, it follows from (2.12) and (2.13) that for $u \in D\left(A_{1}\right)$

$$
|u|_{L^{1}} \leq \sup \left\{\int_{\Omega} u\left(\lambda I-A_{c}\right) v d x: v \in D\left(A_{c}\right),|v|_{L^{\infty}} \leq M|\lambda|^{-1}\right\} .
$$

Using Green's formula we obtain

$$
\int_{\Omega} u A_{c} v d x=\int_{\Omega} v A_{1} u d x
$$

for all $v \in D\left(A_{c}\right)$ and $u \in H^{2}$ with $B u=0$; a density argument implies that this identity holds, moreover, for all $v \in D\left(A_{c}\right)$ and $u \in D\left(A_{1}\right)$.

Thus, for every $u \in D\left(A_{1}\right)$ and $v \in D\left(A_{c}\right)$ with $|v|_{L^{\infty}} \leq M|\lambda|^{-1}$ we have

$$
\begin{aligned}
\left|\int_{\Omega} u\left(\lambda I-A_{c}\right) v d x\right| & =\left|\int_{\Omega}\left(\lambda I-A_{1}\right) u v d x\right| \\
& \leq|v|_{L^{\infty}}\left|\left(\lambda I-A_{1}\right) u\right|_{L^{\prime}} \leq \frac{M}{|\lambda|}\left|\left(\lambda I-A_{1}\right) u\right|_{L^{1}} .
\end{aligned}
$$

Using this in (2.14), it follows that

$$
|u|_{L^{1}} \leq \frac{M}{|\lambda|}\left|\left(\lambda I-A_{1}\right) u\right|_{L^{1}}
$$

Therefore $\lambda I-A_{1}$ is injective and, moreover, since it is closed, its range is closed as well. Since $D\left(A_{c}\right) \subset D\left(A_{1}\right)$ we have that range $\left(\lambda I-A_{1}\right) \supset C(\bar{\Omega})$, for $\lambda \in \Sigma_{\theta}$. This implies range $\left(\lambda I-A_{1}\right)=L^{1}(\Omega)$ for every $\lambda \in \Sigma_{\theta}$ and by $(2.15)$

$$
\left\|\left(\lambda I-A_{1}\right)^{-1}\right\|_{L^{\infty}} \leq \frac{M}{|\lambda|} \text {. }
$$

Since $A_{1}$ is closed and densely defined, this estimate implies that $A_{1}$ generates an analytic semigroup $S_{1}(t)$ on $L^{1}(\Omega)$. Positivity of $S_{1}(t)$ follows from positivity of $S_{c}(t)$ and the fact that sequences converging in the mean contain a subsequence converging almost everywhere. This ends the proof.

Definition 2.1. A pair of functions $\left(v_{1}, v_{2}\right)$ is called a solution of $(2.1)$ if

(i) $\left(v_{1}, v_{2}\right) \in C\left(0, T ; L^{1}(\Omega)\right) \times C^{1}\left(0, T ; L^{1}(\Omega)\right)$,

(ii) $v_{2}$ satisfies $(2.5)$, 
(iii) $v_{1}=\tilde{w}+w$, where $\tilde{w}$ satisfies $(2.6)$, and

(iv) $w$ is the mild solution of (2.4); see (2.16).

In particular, $\left(v_{1}(0), v_{2}(0)\right)=\left(v_{1}^{0}, v_{2}^{0}\right)$.

Theorem 2.1. Assume that (A2) holds and let $T>0$ and $\left(v_{1}^{0}, v_{2}^{0}\right) \in L^{1}(\Omega) \times L^{1}(\Omega)$ be arbitrary. Then there exists a unique solution $\left(w_{1}, v_{2}, \tilde{w}\right) \in C\left(0, T ; L^{1}(\Omega)\right) \times$ $C^{1}\left(0, T ; L^{1}(\Omega)\right) \times C^{1}\left(0, T ; H^{2}(\Omega)\right)$ of $(2.4)-(2.6)$. Consequently $\left(v_{1}, v_{2}\right)=(w+$ $\left.\tilde{w}, v_{2}\right)$ is the unique solution of $(2.1)$ in $C\left(0, T ; L^{1}(\Omega)\right) \times C^{1}\left(0, T ; L^{1}(\Omega)\right)$. Moreover, $\left(v_{1}^{0}, v_{2}^{0}\right) \rightarrow\left(v_{1}\left(\cdot ; v_{1}^{0}, v_{2}^{0}\right), v_{2}\left(\cdot ; v_{1}^{0}, v_{2}^{0}\right)\right)$ is globally Lipschitz continuous from $L^{1}(\Omega) \times$ $L^{1}(\Omega)$ to $C\left(0, T ; L^{1}(\Omega)\right) \times C^{1}\left(0, T ; L^{1}(\Omega)\right)$.

Proof. We use a fixed-point argument to verify the claim. The mild form of (2.4) is given by

$$
w(t)=S_{1}(t) w(0)+\int_{0}^{t} S_{1}(t-s) \frac{\partial}{\partial s} \tilde{w}(s) d s,
$$

which is meaningful for $w(0)=v_{1}^{0}-\tilde{w}\left(0, v_{2}^{0}\right) \in L^{1}(\Omega)$ and $\tilde{w} \in W^{1,1}\left(0, T ; L^{1}(\Omega)\right)$. Moreover, for $v_{2} \in W^{1,1}\left(0, T ; L^{1}(\Omega)\right)$ we determine $\tilde{w} \in W^{1,1}\left(0, T ; H^{2}(\Omega)\right)$ from Lemma 2.2. With $w$ and $\tilde{w}$ thus expressed as functions of $v_{2}$ we solve (2.5). The index in $v_{2}$ is dropped in the subsequent steps.

For $\rho<0$ let $\mathscr{S}=\left\{v \in W_{\rho}^{1,1}\left(0, T ; L^{1}(\Omega)\right) \mid v(0)=v_{2}^{0}\right\}$, where $|v|_{W_{\rho}^{1.1}}=$ $\int_{0}^{T} e^{\rho s}(|v(s)|+|\dot{v}(s)|) d s$ (see [4, pp. 140, 145]). Obviously $\mathscr{S}$ is a closed subset of $W_{\rho}^{1,1}(\Omega)$, and the $W_{\rho}^{1,1}(\Omega)$-norm is equivalent to the usual $W^{1,1}(\Omega)$-norm for every $\rho<0$. We define $\mathscr{T}: \mathscr{S} \rightarrow \mathscr{S}$ by

$$
(\mathscr{T} v)(t)=\exp \left(-a_{2} t\right) v_{2}^{0}+\int_{0}^{t} \exp \left(-a_{2}(t-s)\right) g(w(s, v(s))+\tilde{w}(s, v(s))) d s,
$$

where $t \rightarrow \tilde{w}(t, v(t))$ is the function described in Lemma 2.2 and $t \rightarrow w(t, v(t))$ is given by $(2.16)$. Since

$$
\frac{d}{d t}(\mathscr{T} v)(t)=-a_{2}(\mathscr{T} v)(t)+g(w(t, v(t))+\tilde{w}(t, v(t)))
$$

it follows that in fact $\mathscr{T S} \subset \mathscr{S}$. We show that for $\rho$ appropriately chosen, $\mathscr{T}$ is a contraction on $\mathscr{S}$. Let $v, \bar{v}$ be in $\mathscr{S}$. First observe that with $M=\sup _{[0, T]}\left\|S_{1}(t)\right\|_{L^{\prime}}$ we have from $(2.16)$, and using $w(0, v)=w(0, \bar{v}), \tilde{w}(0, v)=\tilde{w}(0, \bar{v})$,

$$
\begin{aligned}
& \int_{0}^{T} e^{\rho s}(|w(s, v)-w(s, \bar{v})|+|\tilde{w}(s, v)-\tilde{w}(s, \bar{v})| d s \\
& \quad \leq \int_{0}^{T} e^{\rho s}(M+1) \int_{0}^{S}|\dot{\tilde{w}}(\sigma, v)-\dot{\tilde{w}}(\sigma, \bar{v})| d \sigma d s \\
& \quad \leq(M+1) \int_{0}^{T} e^{\rho \sigma}|\dot{\tilde{w}}(\sigma, v)-\dot{\tilde{w}}(\sigma, \bar{v})| \int_{\sigma}^{T} e^{\rho(s-\sigma)} d s d \sigma \\
& \quad \leq \frac{M+1}{|\rho|} \int_{0}^{T}|\dot{\tilde{w}}(\sigma, v)-\dot{\tilde{w}}(\sigma, \bar{v})| d \sigma \leq \frac{C(M+1)}{|\rho|}|v-\bar{v}|_{\mathcal{W}^{\prime} \cdot 1 \cdot\left(0, T ; L^{\prime}(\Omega)\right) .}
\end{aligned}
$$


Let $\bar{a}=\operatorname{ess} \sup _{x}\left(0,-a_{2}(x)\right)$. Then by (A2) and (2.18)

$$
\begin{aligned}
& \int_{0}^{T} e^{\rho t}|(\mathscr{T} v)(t)-(\mathscr{T} \bar{v})(t)|_{L^{\prime}} d t \\
& \quad \leq \int_{0}^{T} e^{\rho t} \int_{0}^{T} \exp (\bar{a}(t-s))\|g\||w(s, v)-w(s, \bar{v})-\tilde{w}(s, v)+\tilde{w}(s, \bar{v})| d s d t \\
& \quad \leq\|g\| \exp (\bar{a} T) T \int_{0}^{T} e^{\rho s}(|w(s, v)-w(s, \bar{v})|+|\tilde{w}(s, v)-\tilde{w}(s, \bar{v})|) d s \\
& \quad \leq C_{3}|\rho|^{-1}|v-\bar{v}|_{W^{\prime .1}\left(0, T ; L^{\prime}(\Omega)\right)},
\end{aligned}
$$

where $C_{3}=\|g\| \exp (\bar{a} T) T C(M+1)$. Moreover, again by $(2.18)$,

$$
\begin{aligned}
\int_{0}^{T} e^{\rho t} & \left|\frac{d}{d t}(\mathscr{T} v-\mathscr{T} \bar{v})(t)\right| d t \\
\leq & \left|a_{2}\right|_{L^{\infty}} C_{3}|\rho|^{-1}|v-\bar{v}|_{W_{p}^{\prime \prime \prime}\left(0, T ; L^{\prime}(\Omega)\right)}+\|g\| \int_{0}^{T} e^{\rho t}(|w(t, v)-w(t, \bar{v})| \\
& \quad+|\tilde{w}(t, v)-\tilde{w}(t, \bar{v})|) d t \\
\leq & \left(\left|a_{2}\right|_{L^{\infty}} C_{3}+\|g\| C(M+1)\right)|\rho|^{-1}|v-\bar{v}|_{W^{\prime \prime .1}\left(0, T ; L^{\prime}(\Omega)\right)} .
\end{aligned}
$$

Thus, for $|\rho|, \rho<0$, sufficiently large, $\mathscr{T}$ is a contraction on $\mathscr{S}$ and there exists a unique fixed point $v_{2} \in W^{1,1}\left(0, T ; L^{1}(\Omega)\right)$ satisfying

$$
\begin{aligned}
& \dot{v}_{2}(t)=-a_{2} v_{2}(t)+g\left(w\left(t, v_{2}\right)+\tilde{w}\left(t, v_{2}\right)\right), \quad \text { a.e. on }(0, T), \\
& v_{2}(0)=v_{2}^{0} .
\end{aligned}
$$

By Lemma 2.2 it follows that $\tilde{w}=\tilde{w}\left(v_{2}\right) \in W^{1,1}\left(0, T ; H^{2}(\Omega)\right)$ and $w \in$ $C\left(0, T ; L^{1}(\Omega)\right)$ by (2.16). Consequently, using the equation for $v_{2}$ and Lemma 2.2 again, we have $v_{2} \in C^{1}\left(0, T ; L^{1}(\Omega)\right)$ and $\tilde{w} \in C^{1}\left(0, T ; H^{2}(\Omega)\right)$. Since $v_{1}=w+\tilde{w}$, we have $v_{1} \in C\left(0, T ; L^{1}(\Omega)\right)$.

The final claim concerning continuous dependence can easily be verified by Gronwall's lemma.

Theorem 2.1 implies that a $C_{0}$-semigroup on $L^{1}(\Omega) \times L^{1}(\Omega)$ can be associated with (2.1). For $\left(v_{1}^{0}, v_{2}^{0}\right) \in L^{1}(\Omega) \times L^{1}(\Omega)$ and $t \geq 0$ let

$$
T_{1}(t)\left(v_{1}^{0}, v_{2}^{0}\right)=\left(v_{1}\left(t ; v_{1}^{0}, v_{2}^{0}\right), v_{2}\left(t ; v_{1}^{0}, v_{2}^{0}\right)\right) .
$$

CoRollary 2.1. Let (A2) hold. Then the family $\left\{T_{1}(t): t \geq 0\right\}$ of operators is a nonlinear $C_{0}$-semigroup, i.e., $T_{1}(0)=I ; T_{1}(t+s)=T_{1}(t) T_{1}(s)$ for $t \geq 0, s \geq 0$, $t \rightarrow T_{1}(t)\left(v_{1}^{0}, v_{2}^{0}\right)$ is continuous from $[0, \infty]$ to $L^{1}(\Omega) \times L^{1}(\Omega)$ and for every $t \geq 0$ there exists $k(t)$ such that

$$
\left|T_{1}(t) z_{1}-T_{1}(t) z_{2}\right|_{L^{1} \times L^{\prime}} \leq k(t)\left|z_{1}-z_{2}\right|_{L^{1} \times L^{1}} \quad \text { for } z_{1}, z_{2} \in L^{1}(\Omega) \times L^{1}(\Omega) .
$$

Proof. The semigroup property $T_{1}(t+s)=T_{1}(t) T_{1}(s)$ is due to the autonomous character of (2.1) and can be verified by means of the following characterization of $v_{2}$ :

$$
\begin{aligned}
v_{2}(t)=\exp \left(-a_{2} t\right) v_{2}^{0}+\int_{0}^{t} \exp \left(-a_{2}(t-s)\right) g\left[S_{1}(s)\left(v_{1}^{0}-A_{1}^{-1} v_{2}^{0}\right)\right. \\
\left.+\int_{0}^{s} S_{1}(s-\sigma) \widetilde{A_{1}^{-1} v_{2}(\sigma)} d \sigma+A_{1}^{-1} v_{2}(s)\right] d s
\end{aligned}
$$


The remaining assertions follow from Theorem 2.1.

THEOREM 2.2. Let $\Omega \subset \mathbf{R}^{n}$ with $n \leq 3$, let (A2) hold and assume that $g: C(\bar{\Omega}) \rightarrow$ $C(\bar{\Omega})$ is globally Lipschitz continuous. Then for every $T>0$ there exists a unique solution $\left(w, v_{2}, \tilde{w}\right) \in C(0, T ; C(\bar{\Omega})) \times C^{1}(0, T ; C(\bar{\Omega})) \times C^{1}\left(0, T ; H^{2}(\Omega)\right)$ of (2.4)(2.6) provided $\left(v_{1}^{0}, v_{2}^{0}\right) \in C(\bar{\Omega}) \times C(\bar{\Omega})$. Consequently, the solution of (2.1) satisfies $\left(v_{1}, v_{2}\right) \in C(0, T ; C(\bar{\Omega})) \times C^{1}(0, T ; C(\bar{\Omega}))$. Moreover, $\left(v_{1}^{0}, v_{2}^{0}\right) \rightarrow T_{c}(t)\left(v_{1}^{0}, v_{2}^{0}\right)=$ $\left(v_{1}\left(\cdot ; v_{1}^{0}, v_{2}^{0}\right), v_{2}\left(\cdot ; v_{1}^{0}, v_{2}^{0}\right)\right)$ is globally Lipschitz continuous from $C(\bar{\Omega}) \times C(\bar{\Omega})$ to $C(0, T ; C(\overline{\mathbf{\Omega}})) \times C(0, T ; C(\overline{\mathbf{\Omega}}))$. Thus $T_{c}(t)\left(v_{1}^{0}, v_{2}^{0}\right)$ is a $C_{0}$-semigroup on $C(\bar{\Omega}) \times C(\overline{\mathbf{\Omega}})$.

Proof. Using the well-known embedding $H^{2}(\Omega) \subset C(\bar{\Omega})$ in dimension not greater than 3 and Lemma 2.3 this result is verified analogously to Theorem 2.1.

Several regularity results can now be obtained easily. We just present the following one, where for $\varphi \in H^{2}(\Omega)$ we put $A \varphi=\Delta \varphi-a_{1} \varphi$.

THEOREM 2.3. In addition to the assumption of Theorem 2.2 let $\left(v_{1}^{0}, v_{2}^{0}\right) \in C(\bar{\Omega}) \times$ $C(\bar{\Omega})$. Then $v=\left(v_{1}, v_{2}\right)$ is a classical solution of $(2.1)$ on $(0, \infty)$ in the sense that $v_{1} \in C(0, T ; C) \cap C^{1}(\varepsilon, T ; C) \cap C\left(\varepsilon, T ; H^{2}\right), v_{2} \in C^{1}(0, T ; C(\bar{\Omega})), A v_{1} \in C(\varepsilon, T ; C(\bar{\Omega}))$, and $B v_{1}(t)=\Phi v_{2}(t)$ on $\Gamma$ for every $t>\varepsilon$ and every pair $\varepsilon, T$ with $0<\varepsilon<T$. If moreover $B v_{1}^{0}=\Phi v_{2}^{0}$ on $\Gamma, v_{1}^{0} \in H^{2}(\Omega)$, and $A v_{1}^{0} \in C(\bar{\Omega})$, then $v$ is a classical solution on $[0, \infty)$, i.e., $\varepsilon$ can be taken equal to 0 .

Proof. From the proof of Theorem 2.1 we have $\tilde{w} \in C^{1}\left(0, T ; H^{2}(\Omega)\right)$ and thus $\tilde{w} \in C^{1}(0, T ; C(\bar{\Omega}))$. Recall that

$$
w(t)=S_{c}(t) w(0)+\int_{0}^{t} S_{c}(t-s) \frac{d}{d s} \tilde{w}(s) d s,
$$

where $w(0)=v_{1}^{0}-\tilde{w}(0) \in C(\bar{\Omega})$. Thus $w$ is the mild solution of $\dot{w}=A_{c} w(t)+$ $d \tilde{w}(t) / d t$. With $d \tilde{w}(t) / d t \in C(0, T ; C(\bar{\Omega}))$ and $A_{c}$ the infinitesimal generator of an analytic semigroup. It is well known [13, p. 110] that $w$ is locally Hölder continuous on $(0, T]$.

By (2.5), Lipschitz continuity of $g$, and the abovementioned properties of $w$ and $\tilde{w}$, local Hölder continuity of $\dot{v}_{2}$ on $(0, T]$ with values in $C(\bar{\Omega})$ follows. Recall that $\dot{\tilde{w}}$ satisfies (2.6) with $v_{2}$ replaced by $\dot{v}_{2}$. Therefore (2.9) of Lemma 2.1 implies local Hölder continuity of $\dot{\tilde{w}}$ on $(0, T]$ with values in $C(\bar{\Omega})$. Now a classical result on regularity of mild solution [13, p. 113] implies that $\dot{w} \in C(\varepsilon, T ; C(\bar{\Omega})), A_{c} w \in C(\varepsilon, T ; C(\bar{\Omega}))$, for every $\varepsilon>0$. In particular, $B w(t)=0$ for $t>0$. Since $v_{1}=w+\tilde{w}$ and $A \tilde{w}=0$, it follows that $\dot{v}_{1} \in C(\varepsilon, T ; C(\bar{\Omega})), A v_{1} \in C(\varepsilon, T ; C(\bar{\Omega})), v_{1} \in C\left(\varepsilon, T ; H^{2}(\bar{\Omega})\right)$, and $B v_{1}(t)=\Phi v_{2}(t)$. Here we used the fact that the embedding of $D\left(A_{c}\right)$ endowed with the graph norm into $H^{2}(\Omega)$ is continuous.

Finally, if $B v_{1}^{0}=\Phi v_{2}^{0}$ on $\Gamma$, then $B w(0)=B v_{1}^{0}-B \tilde{w}(0)=0$ and therefore $w(0)=$ $v_{1}^{0}-w(0) \in D\left(A_{c}\right)$. In this case $w$ and therefore $\dot{v}_{2}$ and $\dot{\tilde{w}}$ are Hölder continuous on $[0, T]$. Thus $\dot{w} \in C(0, T ; C(\bar{\Omega})), A_{c} w \in C(0, T ; C(\bar{\Omega}))$, and the final claim follows.

The final results of this section are concerned with positivity of $T_{1}(t)$ and $T_{c}(t)$. Let $L_{+}^{1}(\Omega)=\left\{\varphi \in L^{1}(\Omega) \mid \varphi(x) \geq 0\right.$ almost everywhere $\}$ and $C_{+}(\bar{\Omega})=\{\varphi \in C(\bar{\Omega}) \mid$ $\varphi(x) \geq 0$, for all $x \in \bar{\Omega}\}$. 
THEOREM 2.4. Let the assumptions of Theorem 2.2 hold and let $\Gamma$ be $C^{3+\alpha}, \alpha \in(0,1)$, smooth, $\beta \in C^{2}(\Gamma), K_{x x} \in L^{\infty},\left(v_{1}^{0}, v_{2}^{0}\right) \in C(\bar{\Omega}) \times C(\bar{\Omega})$. If moreover $K\left(x, x^{\prime}\right) \geq 0$ and $g\left(\mathbf{R}^{+}\right) \subset \mathbf{R}^{+}$, then the semigroups $T_{1}(t)$ and $T_{c}(t)$ are positive with respect to the cones $L_{+}^{1}(\Omega) \times L_{+}^{1}(\Omega)$ and $C_{+}(\bar{\Omega}) \times C_{+}(\bar{\Omega})$.

Proof. Let $\mathscr{M}=\left\{\left(v_{1}^{0}, v_{2}^{0}\right) \in C^{3}(\bar{\Omega}) \cap C_{+}(\bar{\Omega}) \times L_{+}^{1}(\Omega) \mid B v_{1}^{0}=\Phi\left(v_{2}^{0}\right)\right.$ on $\left.\Gamma\right\}$. We first show that $T_{1}(t) \mathscr{M} \subset L_{+}^{1}(\Omega) \times L_{+}^{1}(\Omega)$ and then we extend this inclusion to all of $L_{+}^{1}(\Omega) \times L_{+}^{1}(\Omega)$. For $\left(v_{1}^{0}, v_{2}^{0}\right) \in \mathscr{M}$, the solution $v_{1}$ of $(2.1 \mathrm{a})-(2.1 \mathrm{c})$ has continuous first and second derivatives with respect to $x$ and a continuous first derivative with respect to $t$, i.e., $v_{1}$ is a classical solution in the sense of [11; see pp. 12, 320]. Let $\varphi \in C^{2}(\bar{\Omega})$ be such that $\frac{1}{2} \leq \varphi(x) \leq \sup _{x \in \bar{\Omega}} \varphi(x)=: M,\left.\varphi\right|_{\Gamma}=1,-\partial \varphi /\left.\partial n\right|_{\Gamma}=m$, with the constant $m$ satisfying $\varphi^{-1} m+\beta>0$ on $\bar{\Omega}$; see [11, p. 17]. We further put $\psi(t, x)=v_{1}(t, x) \varphi(x)$ for $(t, x) \in[0, \infty) \times \Omega$. Then $\psi$ is a classical solution (in the sense of [11]) of

$$
\begin{gathered}
\frac{\partial \psi}{\partial t}=-\left(a_{1}+\frac{\Delta \varphi}{\varphi}-\frac{2 \nabla \varphi \nabla \varphi}{\varphi^{2}}\right) \psi+\Delta \psi-\frac{2 \nabla \varphi}{\varphi} \nabla \psi, \\
\psi(0, x)=v_{1}^{0}(x) \varphi(x), \\
\frac{1}{\varphi} \frac{\partial \psi}{\partial n}+\left.\frac{1}{\varphi}\left(\frac{m}{\varphi}+\beta\right) \psi\right|_{\Gamma}=\Phi\left(v_{2}(t)\right), \quad t>0 .
\end{gathered}
$$

Since $(1 / \varphi)(m / \varphi+\beta)>0$ there exists a function $\widehat{C}(t)>0$, depending on $\varphi$, such that

$$
\psi(t, x) \geq \min \left\{0, \min _{S_{1}} \Phi\left(v_{2}\right), \min _{\Omega} v_{1}^{0}\right\} \widehat{C}(t)
$$

[11, Theorem I.2.2], where $S_{t}=[0, t] \times \Omega$. This implies

$$
v_{1}(t, x) \geq \frac{1}{M} \min \left\{0, \min _{S_{t}} \Phi\left(v_{2}\right), \min v_{1}^{0}\right\} \widehat{C}(t)
$$

for all $t \geq 0, x \in \bar{\Omega}$.

Now we restrict the fixed-point operator of Theorem 2.1 to the closed set $\mathscr{S}^{+}=$ $\left\{v_{2} \in W_{\rho}^{1,1}\left(0, T ; L^{1}(\Omega)\right) \mid v_{2}(0)=v_{2}^{0}, \quad v_{2}(t) \in L_{+}^{1}(\Omega)\right\}$. Observe that for $v_{2} \in \mathscr{S}^{+}$ we have $v_{1}(t, x)=w(t, x)+\tilde{w}(t, x) \geq 0$ for $(t, x) \in[0, T] \times \bar{\Omega}$ by (2.21). From the definition of $\mathscr{T}$ it follows that $\mathscr{T S}^{+} \subset \mathscr{S}^{+}$. This implies $v_{2}\left(t, x ; v_{1}^{0}, v_{2}^{0}\right) \geq 0$ for every $t$ and almost every $x \in \Omega$. Employing (2.21) once again, we find $v_{1}\left(t, x ; v_{1}^{0}, v_{2}^{0}\right) \geq 0$ for every $t$ and $x$. It will follow as a consequence of the results of Sec. 3 (see Remark 3.1) that $\mathscr{M}$ is dense in $L_{+}^{1}(\Omega) \times L_{+}^{1}(\Omega)$. Thus for every $\left(v_{1}^{0}, v_{2}^{0}\right) \in L_{+}^{1}(\Omega) \times L_{+}^{1}(\Omega)$ there exists a sequence $v_{1, n}^{0} \in C^{3}(\bar{\Omega}) \cap C_{+}(\bar{\Omega})$ with $B\left(v_{1, n}^{0}\right)=\Phi\left(v_{2}^{0}\right)$ on $\Gamma$ such that $v_{1, n}^{0} \rightarrow v_{1}^{0}$ in $L^{1}(\Omega)$. We know that $v_{1, n}\left(t, x ; v_{1, n}^{0}, v_{2, n}^{0}\right) \geq 0$ for all $t \geq 0$ and $x \in \Omega$. For every $t$ there exists a subsequence $v_{1, n_{k}}\left(t, \cdot ; v_{1, n_{k}}^{0}, v_{2}^{0}\right)$ of $v_{1, n}\left(t, \cdot ; v_{1, n}^{0}, v_{2}^{0}\right)$ converging almost everywhere to $v_{1}\left(t, \cdot ; v_{1}^{0}, v_{2}^{0}\right)$. This implies that $\left(v_{1}\left(t ; v_{1}^{0}, v_{2}^{0}\right), v_{2}\left(t ; v_{1}^{0}, v_{2}^{0}\right)\right) \in L_{+}^{1}(\Omega) \times$ $L_{+}^{1}(\Omega)$ for every $t \geq 0$ and thus $T_{1}(t)$ is a family of positive operators.

Positivity of $T_{c}(t)$ follows from positivity of $T_{1}(t)$. 
3. Remarks concerning the linear model. In this section we consider some aspects of the linear equation

$$
\begin{aligned}
\frac{\partial v_{1}}{\partial t} & =-a_{1} v_{1}+\Delta v_{1}, \\
\frac{\partial v_{2}}{\partial t} & =-a_{2} v_{2}+g v_{1}, \quad \text { for }(t, x) \in(0, \infty) \times \Gamma, \\
\frac{\partial v_{1}}{\partial n}+\beta v_{1} & =\int_{\Omega} K\left(x, x^{\prime}\right) v_{2}\left(t, x^{\prime}\right) d x^{\prime} \quad \text { for }(t, x) \in(0, \infty) \times \Gamma, \\
v_{1}(0, x) & =v_{1}^{0}(x), \quad v_{2}(0, x)=v_{2}^{0}(x),
\end{aligned}
$$

where $g$ is a real-valued function. The following assumptions are made throughout this section:

$$
\begin{aligned}
& a_{1} \in C^{1}(\bar{\Omega}), \quad a_{1} \geq \alpha \geq 0, \\
& a_{2} \in C(\bar{\Omega}), \quad a_{2} \geq 0, \\
& n \leq 3, \\
& \Gamma \text { is } C^{3+\alpha} \text { smooth, } \quad \alpha \in(0,1), \\
& \beta \in C^{1+\alpha}(\Gamma), \quad \beta \geq 0, \\
& g \in C(\bar{\Omega}), \quad K, K_{x} \in L^{\infty}(\Gamma \times \Omega) .
\end{aligned}
$$

By Theorem 2.2 there exists a unique solution $\left(v_{1}, v_{2}\right) \in C(0, T ; C) \times C^{1}(0, T ; C)$ of (3.1) and a linear $C^{0}$-semigroup is generated via

$$
T_{L}(t)\left(v_{1}^{0}, v_{2}^{0}\right)=\left(v_{1}\left(t ; v_{1}^{0}, v_{2}^{0}\right), v_{2}\left(t ; v_{1}^{0}, v_{2}^{0}\right)\right)
$$

We shall frequently write $C$ for $C(\bar{\Omega})$. The additional regularity assumptions on $\Gamma$ and $\beta$ in (A3) over (A2) are used in Theorem 3.2 below.

THEOREM 3.1. Let (A3) holci. The solutions of (3.1) generate a linear $C^{0}$-semigroup $T_{L}(t)$ on $C \times C$. The infinitesimal generator $A_{L}$ of $T_{L}$ is given by

$$
\begin{aligned}
D\left(A_{L}\right) & =\left\{(u, v) \in C \times C \mid u \in H^{2}, \Delta u \in C, B u=\Phi v\right\}, \\
A_{L}(u, v) & =\left(\Delta u-a_{1} v,-a_{2} v+g u\right) .
\end{aligned}
$$

Proof. It only remains to characterize the generator of $T_{L}$ in $C \times C$. We denote it by $\Lambda$ and show that $\Lambda=A_{L}$. This will be accomplished by the core theorem; see [9, Theorem 1.9]. We need to show that

(a) $D\left(A_{L}\right)$ is invariant under $T_{L}(t)$ for $t>0$,

(b) $D\left(A_{L}\right)$ is dense in $C \times C$,

(c) $D\left(A_{L}\right) \subset D(\Lambda)$,

(d) $A_{L}$ is closed.

Observe that (a) and (c) follow from Theorem 2.2. As for density let $(\tilde{u}, \tilde{v}) \in C \times C$. Since $\Phi(\tilde{v}) \in W^{1, \infty}(\Gamma)$, there exists an extension $w$ of $\Phi(\tilde{v})$ from $\Gamma$ to $\Omega$ satisfying $w \in C$ and $B w \mid \Gamma=\Phi(\tilde{v})$ (see, e.g., [15, p. 133]). Since $\tilde{u}-w \in C$ and since $D\left(A_{c}\right)$ is dense in $C$ there exists a sequence $u_{n} \in H^{2}(\Omega)$ satisfying $u_{n} \rightarrow \tilde{u}-w$ in $C$ and $B u_{n}=0$. Thus $\left(u_{n}+w, \tilde{v}\right) \rightarrow(\tilde{u}, \tilde{v})$ in $C$ and $\left(u_{n}+w, \tilde{v}\right) \in D\left(A_{L}\right)$.

To verify closedness of $A_{L}$, let $\left(u_{n}, v_{n}\right) \in D\left(A_{L}\right)$ with $\left(u_{n}, v_{n}\right) \rightarrow(u, v)$ and $\left(\Delta u_{n}-\right.$ $\left.a_{1} u_{n},-a_{2} u_{n}+g v_{n}\right) \rightarrow(x, y)$ in $C \times C$. Since $\left|u_{n}\right|_{H^{2}} \leq \operatorname{const}\left(\left|\Delta u_{n}\right|_{L^{2}}+\left|u_{n}\right|_{L^{2}}\right)[13$, p. 213], it follows that $\left\{u_{n}\right\}$ is bounded in $H^{2}(\Omega)$ and thus there exists a weakly 
convergent subsequence of $u_{n}$, again denoted by $\left\{u_{n}\right\}$, converging strongly in $H^{1}(\Omega)$ to $u$, with $u \in H^{2}(\Omega)$.

Since $-\left(\nabla u_{n}, \nabla \varphi\right)-\left(a_{1} u_{n}, \varphi\right) \rightarrow(x, \varphi)$ for $\varphi \in C_{0}^{\infty}$, we find $(-\nabla u, \nabla \varphi)-\left(a_{1} u, \varphi\right)=$ $(x, \varphi)$ for all $\varphi \in C_{0}^{\infty}$. Thus $\Delta u-a_{1} u=x$. Next observe that $\left|\Delta\left(u_{n}-u\right)\right|_{L^{2}}+\left|u_{n}-u\right|_{L^{2}} \rightarrow$ 0 and thus $u_{n} \rightarrow u$ in $H^{2}$. This implies that $B u_{n} \rightarrow B u$. Since $B u_{n}=\Phi v_{n} \rightarrow \Phi v$ we also have $B u=\Phi v$. Therefore $(u, v) \in D\left(A_{L}\right), A_{L}(u, v)=(x, y)$, and (d) is verified. This ends the proof.

The next objective is to show positivity of $T_{L}$ in $C \times C$. This requires a preliminary result on the positivity of the resolvent of $A_{L}$ and is of independent interest.

Theorem 3.2. Let (A3) hold and let $\left(v_{1}, v_{2}\right) \in C_{+} \times C_{+}, v_{1}^{0} \not \equiv 0, g>0, K \geq 0, K \not \equiv 0$. Then for all real $\omega$ sufficiently large, there exists a unique solution $\left(u_{1}, u_{2}\right)$ of

$$
\begin{gathered}
-\Delta u_{1}+\left(a_{1}+\omega\right) u_{1}=v_{1}, \\
B u_{1}=\Phi v_{2}, \\
\left(a_{2}+\omega\right) u_{2}-g u_{1}=v_{2} .
\end{gathered}
$$

Moreover, $\left(u_{1}, u_{2}\right) \in \operatorname{int} C_{+} \times \operatorname{int} C_{+}$.

Some preliminaries are required before we can prove this theorem. We first recall a general result on the monotonicity of the spectral radius stated in [2]. Let $E$ be a Banach space with an ordering induced by a cone $P$. We denote $\dot{P}=P-\{0\}$ and put int $P$ for the interior of $P$, provided, of course, that it is nonempty. An operator $V: E \rightarrow E$ is called almost strongly positive if $V(P-\operatorname{ker} V) \subset$ int $P$. The spectral radius of a bounded operator $V: E \rightarrow E$ is denoted by $r(V)$.

Lemma 3.1. Let $E$ be a Banach space with an order induced by a cone $P$ with nonempty interior and let $V$ be an almost strongly positive, compact endomorphism of $E$. Suppose in addition that int $P \cap \operatorname{ker} V=\varnothing$ and that $S$ is an almost strongly positive compact endomorphism of $E$ such that $V u-S u \in P-\operatorname{ker} V$ for every $u \in$ int $P$. Then $r(S)<r(V)$.

Lemma 3.2 [2]. Let $E$ and $V$ be as in Lemma 3.1. Then the equation $\lambda u-V u=v$, $v \in \dot{P}$, has for every $\lambda>r(V)$ exactly one solution $u \in \dot{P}$.

Proof of Theorem 3.2. The proof makes use of the technicalities in [2]. Let $A=$ $-\Delta+a_{1}$. Then (3.2) becomes

$$
\begin{gathered}
(A+\omega) u_{1}=v_{1} \quad \text { in } \Omega, \\
B u_{1}=\int_{\Omega} K\left(x, x^{\prime}\right) u_{2}\left(x^{\prime}\right) d x^{\prime}=\Phi\left(u_{2}\right) \quad \text { in } \Gamma, \\
\left(a_{2}+\omega\right) u_{2}=v_{2}+g u_{1} .
\end{gathered}
$$

From the third equation in (3.3) we have (for $\omega$ sufficiently large so that $\omega+a_{2} \in$ int $C_{+}(\bar{\Omega})$ )

$$
u_{2}=\frac{v_{2}}{a_{2}+\omega}+\frac{g}{a_{2}+\omega} u_{1},
$$


and consequently

$$
\begin{gathered}
(A+\omega) u_{1}=v_{1}, \\
B u_{1}=\Phi\left(\frac{v_{2}}{a_{2}+\omega}\right)+\Phi\left(\frac{g}{a_{2}+\omega} u_{1}\right)
\end{gathered}
$$

Let $S_{\omega}: C(\bar{\Omega}) \times C(\Gamma) \rightarrow C(\bar{\Omega})$ be the solution operator associated with

$$
(A+\omega) u_{1}=f, \quad B u_{1}=g,
$$

with $(f, g) \in C(\bar{\Omega}) \times C(\Gamma)$. In [2] it is shown that $S_{\omega}$ is a compact linear operator with

$$
S_{\omega}: C_{+}(\overline{\mathbf{\Omega}}) \times C_{+}(\Gamma)-(0,0) \rightarrow \operatorname{int} C_{+}(\bar{\Omega}) .
$$

Next we rewrite $(3.5)$ as

$$
\sqrt{\omega} u_{1}-S_{\omega}\left(0, \Phi\left(\frac{\sqrt{\omega} g u_{1}}{a_{2}+\omega}\right)\right)=\sqrt{\omega} S_{\omega}\left(v_{1}, \Phi\left(\frac{v_{2}}{a_{2}+\omega}\right)\right) .
$$

It will be convenient to introduce the linear mapping

$$
T_{\omega}: C(\bar{\Omega}) \rightarrow C(\bar{\Omega}) \quad \text { by } T_{\omega} u=S_{\omega}\left(0, \Phi\left(\frac{\sqrt{\omega} g u}{a_{2}+\omega}\right)\right) .
$$

Observe that $T_{\omega}$ is well defined if $\omega+a_{2} \in \operatorname{int} C_{+}(\bar{\Omega})$ and that

$$
T_{\omega}\left(C_{+}(\bar{\Omega})-\operatorname{ker} T_{\omega}\right) \subset \operatorname{int} C_{+}(\bar{\Omega}) .
$$

In particular, $T_{\omega}$ is almost strongly positive and compact in $C(\bar{\Omega})$. We consider (3.7) in the form

$$
\sqrt{\omega} u_{1}-T_{\omega} u_{1}=f_{\omega}
$$

where

$$
f_{\omega}=\sqrt{\omega} S_{\omega}\left(v_{1}, \Phi\left(\frac{v_{2}}{a_{2}+\omega}\right)\right) \in \operatorname{int} C_{+}(\bar{\Omega}) .
$$

Below we shall prove that

$$
\omega^{\prime}>\omega \Rightarrow r\left(T_{\omega^{\prime}}\right)<r\left(T_{\omega}\right)
$$

for all $\omega$ sufficiently large. Lemma 3.2 then implies that there exists a unique solution $u_{1} \in C_{+}(\bar{\Omega})-\{0\}$ of (3.8) for all sufficiently large $\omega$. But $f_{\omega} \in \operatorname{int} C_{+}(\bar{\Omega})$ and by (3.8) we find $u_{1} \in \operatorname{int} C_{+}(\bar{\Omega})$. Since $g>0$ we conclude with $(3.4)$ that $u_{2} \in \operatorname{int} C_{+}(\bar{\Omega})$ as well.

Finally, we verify (3.9) by Lemma 3.1. Let $\omega^{\prime}>\omega$ and $u \in C_{+}(\bar{\Omega})-\operatorname{ker} T_{\omega^{\prime}}$. Then

$$
(A+\omega)\left(T_{\omega} u-T_{\omega^{\prime}} u\right)=\left(\omega^{\prime}-\omega\right) T_{\omega^{\prime}} u \text { in } C(\bar{\Omega})
$$

and

$$
B\left(T_{\omega} u-T_{\omega^{\prime}} u\right)=\Phi\left(\frac{\sqrt{\omega} g u}{a_{2}+\omega}-\frac{\sqrt{\omega^{\prime}} g u}{a_{2}+\omega^{\prime}}\right) .
$$

But $\left(\omega^{\prime}-\omega\right) T_{\omega^{\prime}} u \in$ int $C_{+}(\bar{\Omega})$ and the argument of $\Phi$ in $(3.10)$ is nonnegative for all sufficiently large $\omega$. Therefore $T_{\omega} u-T_{\omega^{\prime}} u \in \operatorname{int} C_{+}(\bar{\Omega})$. Next we show that $T_{\omega} u-T_{\omega^{\prime}} u \in C_{+}(\bar{\Omega})-\operatorname{ker} T_{\omega^{\prime}}$ for $u \in C_{+}(\bar{\Omega})-\operatorname{ker} T_{\omega^{\prime}}$ (and $\omega$ sufficiently large). If this is not the case then $T_{\omega}^{2} u=T_{\omega} T_{\omega^{\prime}} u$. We find that

$$
(A+\omega)\left(T_{\omega}^{2}-T_{\omega} T_{\omega^{\prime}} u\right)=0
$$


and

$$
B\left(T_{\omega}^{2}-T_{\omega} T_{\omega^{\prime}} u\right)=\Phi\left(T_{\omega} u-T_{\omega^{\prime}} u\right) .
$$

Since by assumption $K \geq 0$ and $K \not \equiv 0$ we find $T_{\omega}^{2} u-T_{\omega} T_{\omega^{\prime}} u \in \operatorname{int} C(\bar{\Omega})$, which is a contradiction. Consequently $T_{\omega} u-T_{\omega^{\prime}} u \in C_{+}(\bar{\Omega})-\operatorname{ker} T_{\omega}$ for $u \in C_{+}(\bar{\Omega})-\operatorname{ker} T_{\omega}$ and, in particular, since $K \not \equiv 0$, for every $u \in \operatorname{int} C_{+}(\bar{\Omega})$. Since moreover int $C_{+}(\bar{\Omega}) \cap$ $\operatorname{ker} T_{\omega}=\varnothing$, Lemma 3.1 with $V=T_{\omega}$ and $S=T_{\omega}$ implies (3.9), for all $\omega$ sufficiently large. This ends the proof.

TheOREM 3.3. Let (A3) hold and let $g>0, K \geq 0, K \not \equiv 0$. Then $T_{L}(t)$ is a positivitypreserving semigroup with respect to the cone $C_{+} \times C_{+}$.

Proof. From Theorem 3.2 it follows that for $\left(v_{1}, v_{2}\right) \in C_{+} \times C_{+}$the solution $\left(u_{1}, u_{2}\right)$ of (3.2) satisfies $\left(u_{1}, u_{2}\right) \in C_{+} \times C_{+}$. Alternatively, $\left(\omega-A_{L}\right)^{-1}$ is a positive operator for all $\omega$ sufficiently large. This implies positivity of $T_{L}(t)$ (see, e.g., [10, p. 162]).

Proposition 3.1. Let $T(t)$ be a linear $C_{0}$-semigroup in a Banach space $E$ leaving a closed convex set $F \subset E$ invariant. Then for every $x \in F$ and every $m \in \mathbf{N}$, there exists a sequence $\left\{x_{k}\right\}$ satisfying $x_{k} \in F \cap D\left(A^{m}\right)$ and $x_{k} \rightarrow x$ in $E$. Here $A$ is the infinitesimal generator of $T(t)$.

Proof. Let $t_{k} \rightarrow 0, t_{k} \in \mathbf{R}^{+}$, and define

$$
x_{k}=t_{k}^{-k} \int_{0}^{t_{k}} \cdots \int_{0}^{t_{k}} T\left(s_{1}\right) \cdots T\left(s_{k}\right) x d s_{k} \cdots d s_{1} .
$$

Since the quotient of the value of an integral and the length of the integration path is contained in the convex closure of the values of the integrand [12, p. 25] we find $x_{k} \in F$. Using repeatedly the fact that for every $x \in E, \int_{0}^{t} T(s) d s$ is contained in $D(A)$, it is straightforward to argue that $x_{k} \in D\left(A^{m}\right)$. Finally $x_{k} \rightarrow x$ from wellknown properties of the Bochner integral. These observations imply the claim.

As a corollary to the results of this section we have

Remark 3.1. The set $\mathscr{M}=\left\{\left(v_{1}^{0}, v_{2}^{0}\right) \in C^{3} \cap C_{+} \times L_{+}^{1}: B v_{1}^{0}=\Phi v_{2}^{0}\right.$ on $\left.\Gamma\right\}$ is dense in $L_{+}^{1} \times L_{+}^{1}$. We shall argue density of $\mathscr{M}$ in $C_{+} \times C_{+}(\Omega)$, which implies the claim. Thus let $(u, v) \in C_{+} \times C_{+}$. Then by Theorem 3.1, 3.3, and Proposition 3.1 there exists a sequence $\left(u_{k}, v_{k}\right) \in D\left(A_{L}^{3}\right) \cap C_{+} \times C_{+}$with $\left(u_{k}, v_{k}\right) \rightarrow(u, v)$ in $C \times C$. Since $u_{k} \in D\left(A_{L}^{3}\right)$ we know that $u_{k} \in H^{6}(\Omega)$ and thus $u_{k} \in C^{3}(\Omega)$ [13, pp. 208, 212], and the assertion is verified.

We also point out that Theorem 3.3 is not a consequence of Theorem 2.4, since the proof of Theorem 2.4 requires density of $\mathscr{M}$ in $L_{+}^{1} \times L_{+}^{1}$. This was shown now with the help of Theorem 3.3.

4. Monotonicity and concavity of the solution semigroup. In order to study further the positivity of the solution semigroup we introduce some additional notation. In $\mathbf{R}^{2}$ consider the partial ordering induced by the positive cone $\mathbf{K}=\mathbf{R}_{+} \times \mathbf{R}_{+}$; i.e., if $\xi=\left(\xi_{1}, \xi_{2}\right)^{\mathrm{T}}$ and $\eta=\left(\eta_{1}, \eta_{2}\right)^{\mathrm{T}}$ belong to $\mathbf{R}^{2}$, then $\xi \geq \eta$ means $\xi_{i} \geq \eta_{i}$, for $i=1,2$; $\xi>\eta$ stands for $\xi \geq \eta$ with $\xi \neq \eta ; \xi \gg \eta$ stands for $\xi_{i}>\eta_{i}, i=1,2$ and $\mathbf{R}_{+}=[0, \infty)$. Further, $X=C\left(\bar{\Omega} ; \mathbf{R}^{2}\right)$ is considered as a partially ordered Banach space endowed with the pointwise ordering of $\mathbf{R}^{2}$; i.e., we set $u \leq v$ if $u(x) \leq v(x), u<v$ if 
$u(x)<v(x)$, and $u \ll v$ if $u(x) \ll v(x)$ for all $x \in \bar{\Omega}$, for a pair of elements $u, v$ in $X$. Moreover, we put $X_{+}=\{u \in X \mid u \geq 0\}$. The components of $u$ are denoted by $u_{1}$ and $u_{2}$. A nonlinear operator $F: \operatorname{dom} F \rightarrow X$ with $\operatorname{dom} F=\left\{\left(u_{1}, u_{2}\right) \in X \mid\right.$ $\Delta u_{1}-a_{1} u_{1} \in C(\bar{\Omega}), B u_{1}=\Phi\left(u_{2}\right)$ on $\left.\Gamma\right\}$ is defined by

$$
F\left(u_{1}, u_{2}\right)=\left(F_{1}\left(u_{1}, u_{2}\right), F_{2}\left(u_{1}, u_{2}\right)\right)=\left(\Delta u_{1}-a_{1} u_{1},-a_{2} u_{2}+g\left(u_{1}\right)\right) .
$$

With this notation specified, the system (2.1) can be written formally as

$$
\begin{gathered}
\frac{d}{d t} u(t)=F(\dot{u}(t)), \quad t>0, \\
u(0)=u^{0} .
\end{gathered}
$$

We shall employ the set of functions $Z_{0}=\left\{\varphi:[0, \infty) \times \Omega \rightarrow \mathbf{R}^{2} \mid \varphi_{1}, \varphi_{1, t}, \varphi_{1, x_{i}}, \varphi_{1, x_{i}, x_{j}}\right.$, $i, j=1,2$ continuous on $(0, \infty) \times \Omega$ and $\varphi_{2}, \varphi_{2, t}$ continuous on $\left.[0, \infty) \times \bar{\Omega}\right\}$. We call $v$ a strictly classical solution of $(2.1)$ if $v$ is a classical solution on $[0, \infty)$ in the sense of Theorem 2.3 and $v \in Z_{0}$. Henceforth we put $\mathscr{C}=\left\{\left(v_{1}^{0}, v_{2}^{0}\right)\right.$ is the initial datum of a strictly classical solution $\}$. In Theorem 4.2 we shall require that $v \in \mathscr{C}$ and $\sigma \in(0,1)$ implies $\sigma v \in \mathscr{C}$. Concerning strictly classical solutions we have the following result, which is a direct consequence of [11, Theorem 4.5.3]. Below $H^{k}(\Omega)$, $H^{k}(\Gamma)$, and $H^{1, k}([0, T] \times \Omega)$ stand for the usual Hölder spaces in the variables $x$ and $(t, x)$, respectively; see $[11$, p. 7$]$.

Proposition 4.1. Let (A2) hold, assume that $g: C(\bar{\Omega}) \rightarrow C(\bar{\Omega})$ is globally Lipschitz continuous, and let $\beta \in H^{1+l}(\Gamma)$ and $K \in C^{2}(\Gamma \times \Omega)$. Then for $\left(v_{1}^{0}, v_{2}^{0}\right) \in$ $H^{l+2}(\bar{\Omega}) \times C(\bar{\Omega})$ with $0<l<1$ and $B\left(v_{1}^{0}\right)=\Phi\left(v_{2}^{0}\right)$ on $\Gamma$ it follows that $v=$ $\left.\left(v_{1}\left(\cdot, \cdot ; v_{1}^{0}, v_{2}^{0}\right), v_{2}\left(\cdot, ; v_{1}^{0}, v_{2}^{0}\right)\right) \in H^{(l+2) / 2, l+2}([0, T] \times \bar{\Omega}) \times C^{1,0}([0, T] ; \bar{\Omega})\right)$. Since $T$ is arbitrary, $v$ is a strictly classical solution of (2.1).

We shall make use below of the following technical lemma, which is a modification of $[14 ; 4.24 .1,4.32 .2]$. The gradient of $\varphi, \operatorname{grad} \varphi$, is taken with respect to the $x$ variables.

Lemma 4.1. For two functions $\varphi, \psi \in Z_{0}$ suppose that $\varphi(t) \leq \psi(t)$ for $t>0$, and suppose further that $\varphi_{1}(\bar{t}, \bar{x})=\psi_{1}(\bar{t}, \bar{x}), \operatorname{grad} \varphi_{1}(\bar{t}, \bar{x})=\operatorname{grad} \psi_{1}(\bar{t}, \bar{x})$, the matrix of the second $x$-derivatives of $\psi_{1}-\varphi_{1}$ at $(\bar{t}, \bar{x})$ being nonnegative for a $(\bar{t}, \bar{x}) \in(0, \infty) \times \Omega$, imply $\varphi_{1, t}(\bar{t}, \bar{x})<\psi_{1, t}(\bar{t}, \bar{x})$, and $\varphi_{2}(\bar{t}, \bar{x})=\psi_{2}(\bar{t}, \bar{x})$ implies $\varphi_{2, t}(\bar{t}, \bar{x})<\psi_{2, t}(\bar{t}, \bar{x})$. Then we have the following alternative:

$(\alpha)$ either $\varphi(t, x) \ll \psi(t, x)$ for $(t, x) \in(0, \infty) \times \Omega$,

$(\beta)$ or there exists a maximal $\bar{t} \in[0, \infty)$ such that $\varphi(x, t) \ll \psi(t, x)$ for all $(t, x) \in$ $(0, \bar{t}] \times \Omega$; i.e., for $i=1$ or 2 there exists a sequence $\left(t_{k}, x_{k}\right) \in(0, \infty) \times \Omega, t_{k}>\bar{t}$ with $\left(t_{k}, x_{k}\right) \rightarrow(\bar{t}, \bar{x}), \bar{x} \in \Gamma$, and $\varphi_{i}\left(t_{k}, x_{k}\right) \geq \psi_{i}\left(t_{k}, x_{k}\right)$.

THEOREM 4.1. Let (A2) hold, let $K \geq 0$, and assume that

(H1) $u$ and $v$ are strictly classical solutions of (4.1),

(H2) $g: \mathbf{R} \rightarrow \mathbf{R}$ satisfies

(i) $g(0)=0$,

(ii) if $z_{1}<z_{2}$ then $g\left(z_{1}\right)<g\left(z_{2}\right)$, 
(iii) $g$ is twice continuously differentiable on $(-\infty, \infty)$ with $0<g^{\prime}(0), g^{\prime \prime}(z)<0$ on $(0, \infty)$, and $g^{\prime \prime}(z)>0$ on $(-\infty, 0)$,

(H3) either $\beta(x) \geq \underline{\beta}>0$ or $K\left(x, x^{\prime}\right) \geq \underline{K}>0$ or there exists a function $h:(0, \infty) \times$ $\bar{\Omega} \rightarrow \mathbf{R}$ which is continuous with continuous first- and second-order derivatives and $\partial h / \partial n>0$ on $(0, \infty) \times \Gamma$.

If moreover

$$
\begin{gathered}
\frac{d}{d t} u(t)-F u(t) \leq \frac{d}{d t} v(t)-F v(t) \quad \text { for all } t>0, \\
\frac{\partial u_{1}}{\partial n}(t, x)+\beta(x) u_{1}(t, x)-\Phi\left(u_{2}\right)(t, x) \\
\leq \frac{\partial v_{1}}{\partial n}(t, x)+\beta(x) v_{1}(t, x)-\Phi\left(v_{2}\right)(t, x) \text { in }(0, \infty) \times \partial \Omega,
\end{gathered}
$$

and

$$
u(0) \leq v(0) \text { on } \bar{\Omega},
$$

then

$$
u(t) \leq v(t) \text { for all } t \geq 0 .
$$

If in addition $u\left(0, x^{*}\right) \neq v\left(0, x^{*}\right)$ for some $x^{*} \in \Omega, d u(t) / d t \leq F u(t), d v(t) / d t \geq$ $F(v(t))$, and $K\left(x, x^{\prime}\right) \geq \underline{K}>0$, then

$$
u(t) \ll v(t) \text { for all } t>0 \text { on } \bar{\Omega} \text {. }
$$

REMARK 4.1. The last hypothesis in (H3) is satisfied if $\Omega$ is convex for example (see [14, p. 249]).

REMARK 4.2. We have the following immediate consequences of $(\mathrm{H} 2)$ :

(i) $g^{\prime}$ is strictly decreasing on $(0, \infty)$, strictly increasing on $(-\infty, 0)$ with $\max _{x \in \mathbf{R}} g^{\prime}(x)=g^{\prime}(0)$;

(ii) $g$ is globally Lipschitz continuous on $(-\infty, \infty)$ with Lipschitz constant $g^{\prime}(0)$;

(iii) for all $\sigma \in(0,1)$ and for all $z>0$ we have $\sigma g(z)<g(\sigma z)$;

(iv) for all $R$ there exists $k_{R}$ such that $-R \leq z_{1} \leq z_{2} \leq R$ implies $g\left(z_{2}\right)-g\left(z_{1}\right) \geq$ $k_{R}\left(z_{2}-z_{1}\right)$.

Proof of Theorem 4.1. The proof will be given in three steps and closely follows techniques described in [14].

Step 1. If in (4.2)-(4.4), the " $\leq$ " estimate is replaced by "«," then this is denoted by $\left(4.2^{\prime}\right)-\left(4.4^{\prime}\right)$ and implies $u(t) \ll v(t)$ for $t \geq 0$. We argue similarly to the proof of $[14,4.32 .4$, p. 258]. First note that Lemma 4.1 is applicable. For assume that at some $\bar{t}, \bar{x} \in(0, \infty) \times \Omega$ we have $u_{1}=v_{1}, u_{2} \leq v_{2}, u_{1, x x} \leq v_{1, x x}$, and $u_{1, y y} \leq v_{1, y y}$. By $\left(4.2^{\prime}\right)$ we have

$$
u_{1, t}+a u_{1}-\Delta u_{1}<v_{1, t}+a_{1} v_{1}-\Delta v_{1}
$$

at $(\bar{t}, \bar{x})$ and therefore $u_{1, l}<v_{1, t}+\Delta u_{1}-\Delta v_{1} \leq v_{1, l}$. The case $u_{1} \leq v_{1}$ and $u_{2}=v_{2}$ at $\bar{t}, \bar{x}$ is handled analogously. Next we show that $(\beta)$ in Lemma 4.1 cannot occur.

Let $(\bar{t}, \bar{x})$ with $\bar{x} \in \Gamma$ be defined as in $(\beta)$; in particular, $u_{i}(\bar{t}, \bar{x})=v_{i}(\bar{t}, \bar{x})$ for $i=1$ or 2 . Since $u(0) \ll v(0)$ on $\bar{\Omega}$ by $\left(4.4^{\prime}\right)$ it follows that $\bar{t}>0$. First we consider the case $i=1$ and observe that by $\left(4.3^{\prime}\right)$

$$
\frac{\partial u_{1}(\bar{t}, \bar{x})}{\partial n}+\beta(\bar{x}) u_{1}(\bar{t}, \bar{x})-\Phi\left(u_{2}(\bar{t})\right)<\frac{\partial v_{1}}{\partial n}(\bar{t}, \bar{x})+\beta(\bar{x}) v_{1}(\bar{t}, \bar{x})-\Phi\left(v_{2}(\bar{t})\right),
$$


and thus

$$
\frac{\partial u_{1}(\bar{t}, \bar{x})}{\partial n}<\frac{\partial v_{1}(\bar{t}, \bar{x})}{\partial n}
$$

This contradicts the fact that due to $v_{1}(\bar{t}, \bar{x})=u_{1}(\bar{t}, \bar{x})$ and $v_{1}(\bar{t}, x)-u_{1}(\bar{t}, x)>0$, for $x \in \Omega$, we have $\partial u_{1}(\bar{t}, \bar{x}) / \partial n \geq \partial v_{1}(\bar{t}, \bar{x}) / \partial n$; compare [14, pp. 247, 258]. Next assume that $u_{2}(\bar{t}, \bar{x})=v_{2}(\bar{t}, \bar{x})$ with $(\bar{t}, \bar{x})$ chosen as in $(\beta)$ of Lemma 4.1. Then $u_{2}(t, \bar{x}) \leq v_{2}(t, \bar{x})$ for all $t \in[0, \bar{t})$ and

$$
\frac{\partial}{\partial t} u_{2}(\bar{t}, \bar{x}) \geq \frac{\partial}{\partial t} v_{2}(\bar{t}, \bar{x})
$$

Since $g$ is monotonically increasing this implies

$$
\frac{\partial}{\partial t} u_{2}(\bar{t}, \bar{x})+a_{2}(\bar{x}) u_{2}(\bar{t}, \bar{x})-g\left(u_{1}(\bar{t}, \bar{x})\right) \geq \frac{\partial}{\partial t} v_{2}(\bar{t}, \bar{x})+a_{2}(\bar{x}) v_{2}(\bar{t}, \bar{x})-g\left(v_{1}(\bar{t}, \bar{x})\right) .
$$

This contradicts $\left(4.2^{\prime}\right)$ and thus $(\alpha)$ must hold. We have already seen that $u \ll v$ on $\Gamma$ and thus $u \ll v$ on $[0, \infty) \times \bar{\Omega}$.

Step 2. We now prove the first part of the theorem. Let us consider the case where one of the first two "or" conditions in (H3) holds. We define for $\lambda>0$ and with $M=\max \left(g^{\prime}(0), 2\left|a_{2}\right|_{C}\right)$

$$
z=\left(\begin{array}{c}
z_{1} \\
z_{2}
\end{array}\right)=\left(\begin{array}{c}
v_{1}+\lambda e^{M t} \\
v_{2}+\lambda e^{2 M t}
\end{array}\right) .
$$

By using the result of Step 1 we will show that $z(t) \gg u(t)$ for every $t>0$. We demonstrate $\left(4.2^{\prime}\right)$ and $\left(4.3^{\prime}\right)$ with $v$ replaced by $z$ :

$$
z_{1, t}+a_{1} z_{1}-\Delta z_{1}=v_{1, t}+\lambda M e^{M t}+a_{1} v_{1}+a_{1} \lambda e^{M t}-\Delta v_{1}>u_{1, t}+a_{1} u_{1}-\Delta u_{1} .
$$

Similarly, since by (4.2) $v_{2, t}+a_{2} v_{2} \geq u_{2, t}+a_{2} u_{2}+g\left(v_{1}\right)-g\left(u_{1}\right)$ we have

$$
\begin{aligned}
z_{2, t}+a_{2} z_{2}-g\left(z_{1}\right) & \geq 2 M \lambda e^{2 M t}+v_{2, t}+a_{2} v_{2}+a_{2} \lambda e^{2 M t}-g\left(z_{1}\right) \\
& \geq \frac{3}{2} M \lambda e^{2 M t}+u_{2, t}+a_{2} u_{2}+g\left(v_{1}\right)-g\left(z_{1}\right)-g\left(u_{1}\right) \\
& \geq u_{2, t}+a_{2} u_{2}-g\left(u_{1}\right)+\frac{3}{2} M \lambda e^{2 M t}-M \lambda e^{M t} \\
& >u_{2, t}+a_{2} u_{2}-g\left(u_{1}\right) .
\end{aligned}
$$

Next, observe that (4.3) implies

$$
\frac{\partial u_{1}}{\partial n}+\beta u_{1} \leq \frac{\partial v_{1}}{\partial n}+\beta v_{1}+\Phi\left(u_{2}-v_{2}\right)
$$

Let $z_{1}(\bar{t}, \bar{x})=u_{1}(\bar{t}, \bar{x})$ with $\bar{t}, \bar{x}$ as in $(\beta)$. Then

$$
\begin{aligned}
\frac{\partial u_{1}}{\partial n} & \leq \frac{\partial v_{1}}{\partial n}+\beta\left(v_{1}-u_{1}\right)+\Phi\left(u_{2}-v_{2}\right) \\
& =\frac{\partial z_{1}}{\partial n}+\beta\left(z_{1}-\lambda e^{M t}-u_{1}\right)+\Phi\left(u_{2}-v_{2}\right) \\
& =\frac{\partial z_{1}}{\partial n}-\beta \lambda e^{M t}+\Phi\left(u_{2}-v_{2}\right)<\frac{\partial z_{1}}{\partial n},
\end{aligned}
$$

at $(\bar{t}, \bar{x})$ for all $\lambda$ sufficiently small, if $\beta>0$ on $\partial \Omega$ or $K>0$ on $\partial \Omega \times \Omega$. Thus (4.5) holds with $v_{1}$ replaced by $z_{1}$. Case $(\beta)$ of Lemma 4.1 can now be excluded with the 
same arguments as in Step 1 and we have $u(t, x) \ll z(t, x)$ for $(t, x) \in[0, \infty) \times \Omega$. Finally, under the third alternative (H3) we define

$$
z_{1}=v_{1}+\lambda e^{\widehat{M} t}+\lambda h, \quad z_{2}=v_{2}+\lambda e^{\mu \widehat{M} t},
$$

where we pick $\mu>3, \widehat{M}>\max \left(M+1,|\Delta h|_{L^{\infty}(\Omega)}\right)$, and assume without loss of generality that $|h|_{L^{\infty}} \leq 1$. With $\left(z_{1}, z_{2}\right)$ thus defined, estimates analogous to those above yield $u(t, x) \ll z(t, x)$ for $(t, x) \in[0, \infty) \times \bar{\Omega}$.

Step 3. If $u_{1}\left(0, x^{*}\right)>v_{1}\left(0, x^{*}\right)$ then $u_{1}(t, x)<v_{1}(t, x)$ for all $(t, x) \in(0, \infty) \times \Omega$; see [3]. Using this inequality together with (2.1b) and Remark 4.2(iv), we obtain $u_{2}(t, x)<v_{2}(t, x)$ for $(t, x) \in(0, \infty) \times \Omega$. Next, employing the boundary condition and the fact that $K\left(x, x^{\prime}\right) \geq \underline{K}$ we find that $u_{1}(t, x)<v_{1}(t, x)$ for all $(t, x) \in(0, \infty) \times \Gamma$. (We observe that a weaker assumption on the nonnegativity of $K$ would be sufficient here: $K \geq 0$ and for every $x^{\prime} \in \Gamma$ there exists $\tilde{x}=\tilde{x}\left(x^{\prime}\right) \in \Omega$ such that $K\left(\tilde{x}, x^{\prime}\right)>0$.) Using (2.1b) once again, we argue that $u_{2}(t, x)<v_{2}(t, x)$ for $(t, x) \in(0, \infty) \times \Gamma$. Summarizing the argument up to now, we have that $u_{1}\left(0, x^{*}\right)<v_{1}\left(0, x^{*}\right)$ implies $u \ll v$ on $(0, \infty) \times \bar{\Omega}$.

Next let $u_{2}\left(0, x^{*}\right)<v_{2}\left(0, x^{*}\right)$. By $(2.1 \mathrm{~b})$ therefore $v_{2}\left(t, x^{*}\right)>u_{2}\left(t, x^{*}\right)$ for all $t>0$. Moreover, for every $T>0$ there exists a neighborhood $U\left(x^{*}\right)$ of $x^{*}$ such that $v_{2}(t, x)>u_{2}(t, x)$ for all $(t, x) \in[0, T] \times U\left(x^{*}\right)$. Since $v_{2} \geq u_{2}$ on $[0, T] \times \bar{\Omega}$, this implies $\Phi\left(v_{2}-u_{2}\right)\left(t, x^{\prime}\right)>0$ for every $\left(t, x^{\prime}\right) \in(0, T] \times \Gamma$. We know that $u_{1} \leq v_{1}$ on $[0, \infty) \times \bar{\Omega}$. Now assume that $u_{1}(\tilde{t}, x)=v_{1}(\tilde{t}, x)$ for some $\tilde{t} \in(0, T]$ and all $x \in \Omega$. Then by (4.3) we have $\Phi\left(u_{2}\right)(\tilde{t}, x)=\Phi\left(v_{2}\right)(\tilde{t}, x)$ for all $x \in \Gamma$. This cannot occur, and thus for every $t \in(0, T]$ there exists at least one $x^{*}$ such that $u_{1}\left(t, x^{*}\right)<v_{1}\left(t, x^{*}\right)$. (Observe that for this argument it would suffice that there exists some $\tilde{x}^{\prime} \in \Gamma$ and $\underline{K}\left(\tilde{x}^{\prime}\right)>0$ such that $K\left(x, \tilde{x}^{\prime}\right) \geq \underline{K}\left(\tilde{x}^{\prime}\right)$.) Using the result in [3] once again, we have $u_{1}(t, x)<v_{1}(t, x)$ for $(t, x) \in(0, T] \times \Omega$ and subsequently, arguing as in the first part of the proof, $u \ll v$ on $(0, T] \times \bar{\Omega}$. Since $T$ was arbitrary this implies the result.

REMARK 4.3. As indicated in the proof, Theorem 4.1 remains correct with the positivity assumption on $K$ replaced by (a) $K \geq 0$, (b) for all $x^{\prime} \in \Gamma$ there exists $\tilde{x}\left(x^{\prime}\right) \in \Omega$ such that $K\left(\tilde{x}, x^{\prime}\right)>0$, and (c) there exists $\tilde{x}^{\prime} \in \Gamma$ and $K\left(\tilde{x}^{\prime}\right)>0$ such that $K\left(x, \tilde{x}^{\prime}\right) \geq K\left(\tilde{x}^{\prime}\right)$ for all $x \in \Omega$.

Theorem 4.1 implies in particular monotonicity results for the solution semigroup associated with (2.1). We shall henceforth drop the indices $c$ and 1 in the notation of $T(t)$.

Under the assumptions of Proposition 4.1 and Theorem 4.1, a density argument similar to that in the proof of Theorem 2.4 (see also Remark 3.1) implies:

$$
\begin{aligned}
& \text { for all } u^{0}, v^{0} \in L^{1}(\Omega) \times L^{1}(\Omega) \text { with } u^{0} \leq v^{0} \text { it follows that } \\
& T(t) u^{0} \leq T(t) v^{0} \text { for all } t \geq 0 .
\end{aligned}
$$

For a classical solution Theorem 4.1 implies moreover

$$
\begin{aligned}
& \text { if } u^{0}, v^{0} \in \mathscr{C} \times \mathscr{C} \text { with } u^{0} \leq v^{0}, u^{0} \neq v^{0} \text {, then } \\
& T(t) u^{0} \ll T(t) v^{0} \text { for all } t>0 ;
\end{aligned}
$$

in particular,

$$
\text { if } 0 \neq u^{0} \in \mathscr{C} \cap X_{+} \text {, then } 0 \ll T(t) u^{0} \text { for all } t>0 \text {. }
$$


Another important result is related to the concavity of the solution operator $T(t)$ on $\mathscr{C}_{+}$.

THEOREM 4.2. Let (A2), (H2) hold and let $K\left(x, x^{\prime}\right) \geq \underline{K}>0$. Then for every $v^{0} \in \mathscr{C} \cap$ $X_{+}$with $v^{0} \neq 0$ for every $\sigma \in(0,1)$ and $t>0$ there exists a constant $\alpha=\alpha\left(v^{0}, t, \sigma\right)>0$ such that $T(t) \sigma v^{0} \geq(1+\alpha) \sigma T(t) v^{0}$.

Proof. We set $\chi(t)=T(t) \sigma v^{0}-\sigma T(t) v^{0}$. Note that $\chi \in Z_{0}$ and $B \chi_{1}(t)=\Phi\left(\chi_{2}(t)\right)$. Moreover, for every $t>0$

$$
\frac{d}{d t} \chi(t)=F\left(T(t) \sigma v^{0}\right)-\sigma F\left(T(t) v^{0}\right) .
$$

By Remark 4.2(iii) and since $T(t) v^{0} \gg 0$ for $t>0$ by Theorem 4.1 we obtain $\partial \chi(t) / \partial t>F\left(T(t) \sigma v^{0}\right)-F\left(\sigma T(t) v^{0}\right)$ for $t>0$ and $x \in \bar{\Omega}$, with equality in the first coordinate. This further implies by the mean value theorem that

$$
\begin{aligned}
\frac{\partial}{\partial t} \chi(t, x) & >\left(\begin{array}{cc}
-a_{1}+\Delta & 0 \\
0 & -a_{2}
\end{array}\right) \chi(t, x)+\left(\begin{array}{c}
0 \\
g\left(\left(T(t) \sigma v^{0}\right)_{1}(x)\right)-g\left(\left(\sigma T(t) v^{0}\right)_{1}(x)\right)
\end{array}\right) \\
& \geq\left(\begin{array}{cc}
-a_{1}+\Delta & 0 \\
0 & -a_{2}
\end{array}\right) \chi(t, x)+\left(\begin{array}{c}
0 \\
g^{\prime}(\xi(t, x)) \chi_{1}(t, x)
\end{array}\right)
\end{aligned}
$$

for $(t, x) \in(0, \infty) \times \bar{\Omega}$, again with equality in the first component. Note that $(t, x) \rightarrow$ $g^{\prime}(\xi(t, x))$ is continuous and that $g^{\prime}(\xi(t, x)) \geq 0$. With minor changes in the proof of Theorem 4.1 due to the term $g^{\prime}(\xi(t, x)) \chi(t, x)$ in (4.9), one can show that $\chi(t) \geq 0$ for every $t \geq 0$.

Using the second component of (4.9) once again, we have

$$
\frac{\partial}{\partial t} \chi_{2}(t)>-a_{2} \chi_{2}(t), \quad \chi_{2}(0)=0,
$$

for all $t>0$. This implies $\chi_{2}(t)>0$ for $t>0$. Now assume that $\chi_{1}(\bar{t}, x)=0$ for all $x \in \Omega$ and some $\bar{t}>0$. Then $B \chi_{1}(\bar{t}, x)=\partial \chi_{1}(\bar{t}, x) / \partial n+\beta(x) \chi_{1}(\bar{t}, x) \leq 0$ for all $x \in \Gamma$; on the other hand, $B \chi_{1}(\bar{t}, x)=\Phi\left(\chi_{2}(\bar{t})\right)(x)$ on $\Gamma$, which is impossible. Thus for every $t>0$ there exists some $x \in \Omega$ such that $\chi_{1}(t, x)>0$. As before, this implies $\chi_{1}(t, x)>0$ for all $(t, x) \in(0, \infty) \times \Omega$ [3]. Using the boundary condition once more, we finally obtain $\chi_{1}>0$ on $(0, \infty) \times \bar{\Omega}$. This ends the proof.

Acknowledgment. Work performed with partial support by the Fonds zur Förderung der Wissenschaftlichen Forschung, Austria, S 3206. V. C. also acknowledges support by the CNR-Italy: Special Program "Control of Infectious Diseases."

\section{REFERENCES}

[1] H. Amann, Dual semigroups and second order linear elliptic boundary value problems, Israel J. Math. 45, 225-254 (1983)

[2] H. Amann, Nonlinear elliptic equations with nonlinear boundary conditions, New Developments in Differential Equations, W. Eckhaus (Editor), North-Holland Math. Studies 21, 43-63 (1976)

[3] P. Besala, An extension of the strong maximum principle for parabolic equations, Bull. Acad. Polon. Sci. Ser. Math. Astronom. Phys. 11, 1003-1006 (1971)

[4] H. Brezis, Operateurs maximaux monotones, North-Holland, Amsterdam, 1973

[5] V. Capasso and K. Kunisch, A reaction-diffusion system modelling man-environment epidemics, Ann. of Differential Equations 1, 1-12 (1985) 
[6] V. Capasso and L. Maddalena, Convergence to equilibrium states for a reaction-diffusion system modelling the spatial spread of a class of bacterial and viral diseases, J. Math. Biol. 13, 173-184 (1981)

[7] V. Capasso and L. Maddalena, On a degenerate nonlinear diffusion problem with boundary feedback, Appl. Analysis 24, 256-298 (1987)

[8] V. Capasso and S. L. Paveri-Fontana, A mathematical model for the 1973 cholera epidemic in the European Mediterranean region, Rev. Epidem. et Santè Publ. 27, 121-132 (1979) (Errata, ibid. 28, $330,1980)$

[9] E. B. Davies, One parameter semigroups, Academic Press, London, 1980

[10] H. O. Fattorini, The Cauchy problem, Encyclopedia of Mathematics and Its Applications, AddisonWesley, Reading, Mass., 1983

[11] O. A. Ladyženskaja, V. A. Solonnikov, and N. N. Ural'ceva, Linear and quasilinear equations of parabolic type, Translations Amer. Math. Soc., Providence, 1968

[12] R. M. Martin, Nonlinear operators and differential equations in Banach spaces, John Wiley \& Sons, New York, 1976

[13] A. Pazy, Semigroups of linear operators and applications to parabolic differential equations, SpringerVerlag, Berlin, 1983

[14] W. Walter, Differential and integral inequalities, Springer-Verlag, Berlin, 1970

[15] J. Wloka, Partielle Differentialgleichungen, Teubner, Stuttgart, 1982

[16] V. Arnautu, V. Barbu, and V. Capasso, Controlling the spread of a class of epidemics, submitted to Appl. Math. and Optimization

[17] K. Kunisch and H. Schelch, Parameter estimation in a special reaction-diffusion system modelling man-environment diseases, to appear in J. Math. Biology

[18] H. Schelch, Parameter estimation in a special reaction-diffusion system modelling man-environment diseases specific for the Mediterranean region, Thesis, Technical University of Graz (in German) 